\title{
A UNIFIED APPROACH FOR LITTLEWOOD-PALEY DECOMPOSITION OF ABSTRACT BESOV SPACES
}

\author{
AZITA MAYELI \\ (Communicated by Alexander Iosevich)
}

\begin{abstract}
We apply spectral theoretic methods to obtain a Littlewood-Paley decomposition of abstract inhomogeneous Besov spaces in terms of "smooth" and "bandlimited" functions. Well-known decompositions in several contexts are shown as special examples and are unified under the spectral theoretic approach.
\end{abstract}

\section{INTRODUCTION}

Besov spaces appear in many subfields of analysis and applied mathematics. In the classical setting, the Besov space $B_{q}^{\alpha}\left(L^{p}\right)$ is the set of functions in $L^{p}$ with smoothness degree $\alpha$ and (quasi)norm controlled by $q$. There are two types of definitions for these spaces. One type uses Fourier transform (for example, see [18]), and the other uses modulus of continuity. The Besov spaces defined by modulus of continuity are more practical in many areas of analysis, for example, in approximation theory. (See [4.)

To understand the structure of the Besov spaces for application purposes, it is natural to decompose a Besov function into simple building blocks and hereby to reduce the study of functions to the study of only the elements in the decomposition. Wavelet and frame theory have been very useful tools to achieve this goal. A unified characterization of Besov spaces in terms of atomic decomposition using the group representation theoretic approach was given by Feichtinger and Gröchenig ( $\underline{5}])$. In the classical level, this kind of decomposition using the spectral theoretic approach was proved in [8]. New results in this direction in the context of Lie groups and homogeneous manifolds were recently published in [2]-3], 6, 7], and [9]-13. In 10, 11, the authors constructed continuous and time-frequency localized wavelets and applied them to the classification of Besov spaces on the compact Riemannian manifolds.

The purpose of the present paper is to describe abstract Besov spaces $B_{q}^{\alpha}(\mathcal{H})$ when $L^{p}$ is replaced by an abstract Hilbert space $\mathcal{H}$. Then the other well-known descriptions of $B_{q}^{\alpha}\left(L^{2}\right)$ will be considered as examples of our theory. The abstract Besov spaces were introduced using modulus of continuity, for example, by Lions in [17]. We use the definition in [17] and establish our results by developing a

Received by the editors February 7, 2014.

2010 Mathematics Subject Classification. Primary 43X46; Secondary 41xxx.

Key words and phrases. Inhomogeneous Besov space, Paley-Wiener space, Littlewood-Paley decomposition.

The research was supported by The Professional Staff Congress-City University of New York Grant. 
connection between "frequency content" of vectors in $\mathcal{H}$ and their "smoothness". We show that a vector (function) belongs to $B_{q}^{\alpha}(\mathcal{H})$ if and only if the sequence of its "filtered" versions satisfies some certain rate of norm convergence in $l^{q}$. By this, we can identify the Besov space $B_{q}^{\alpha}(\mathcal{H})$ with the sequence space $l^{q}$, similar to the identification of a function with its Fourier coefficients. While the idea behind such identification is simple, the proofs are technical and difficulties arise when $L^{2}$ is replaced by an abstract Hilbert space. The main result of this paper appears in Theorem 3.1 and its proof is given in Section 4

\section{Preliminaries}

Let $\mathcal{H}$ be a Hilbert space and $A$ be any self-adjoint positive definite operator in $\mathcal{H}$ whose domain is dense in $\mathcal{H}$. The domain of $A$ contains all functions $f \in \mathcal{H}$ for which $\|A f\|$ is finite. Let $u: \mathbb{R}^{+} \rightarrow \mathbb{R}$ be a positive function such that $u \in C^{\infty}\left(\mathbb{R}^{+}\right)$, $u^{(n)}$ decays rapidly at infinity for all $n \in \mathbb{N}$, and $\lim _{\xi \rightarrow 0^{+}} u^{(n)}(\xi)$ exists. Therefore, $u$ is a Schwartz function on $\mathbb{R}^{+}$. Moreover, we assume that $u(s+t)=u(s) u(t)$ and $u(0)=1$. For $t>0$, we define the operator $T_{t}=u_{t}(A)=u(t A)$ mapping $\mathcal{H}$ to $\mathcal{H}$. Then $\left\{T_{t}\right\}_{t>0}$ satisfies the semigroup axioms $T_{t+s}=T_{t} T_{s}$ and $T_{0}=$ identity. Let $M:=\|u\|_{\infty}$ denote the uniform norm of $u$. Then $M<\infty$ and we have the following:

(1) $\lim _{t \rightarrow 0^{+}} T_{t} f=f$ for all $f \in \mathcal{H}$,

(2) $T_{t}$ is symmetry (self-adjoint), and

(3) $\left\|T_{t} f\right\| \leq M\|f\|$ for all $f \in \mathcal{H}$.

Under the construction of the semigroup $\left\{T_{t}\right\}$, the operator $A$ is the infinitesimal generator of semigroup $\left\{T_{t}\right\}_{t>0}$. That means, in the Hilbert space norm

$$
\lim _{t \rightarrow 0^{+}}\left\|\frac{T_{t} f-f}{t}-A f\right\|=0 .
$$

We define the modulus of continuity for $0<q \leq \infty, \alpha>0$, and $r \in \mathbb{N}$ with $r \geq \alpha$, by

$$
\Omega_{r}(s, f)=\sup _{0<\tau \leq s}\left\|\left(I-T_{\tau}\right)^{r} f\right\| .
$$

Following [17, the inhomogeneous abstract Besov space $B_{q}^{\alpha}:=B_{q}^{\alpha}(\mathcal{H})$, associated to $\mathcal{H}$ and $A$, contains all functions $f \in \mathcal{H}$ for which

$$
\int_{0}^{1}\left(s^{-\alpha} \Omega_{r}(s, f)\right)^{q} \frac{d s}{s}<\infty .
$$

The space $B_{q}^{\alpha}, 1 \leq q<\infty$, becomes a Banach space with the norm

$$
\|f\|+\left(\int_{0}^{1}\left(s^{-\alpha} \Omega_{r}(s, f)\right)^{q} \frac{d s}{s}\right)^{1 / q} .
$$

We use the standard convention for the definition of norm when $q=\infty$.

Remark. Notice that the Besov norm does not depend on $r$ due to the monotonicity of the modulus of continuity. Therefore, for fixed $q$ and $\alpha$, all definitions of Besov spaces in terms of modulus of continuity are equivalent, so they are independent of the choice of $r$. 
By the definition of the Besov spaces, it is trivial that the following inclusions hold:

$$
\mathcal{D}(A) \subset B_{q}^{\alpha} \subset \mathcal{H}
$$

where $\mathcal{D}(A)$ is the domain of the operator $A$ and is dense in $\mathcal{H}$ under the assumptions. Also, note that the space $\mathcal{D}(A)$ is a Banach space with the norm $\|f\|+\|A f\|$. (See, for example, [14.)

By the spectral theory, the operator $A$ has a representation

$$
A=\int_{0}^{\infty} \xi d P_{\xi},
$$

where $d P_{\xi}$ is a spectral measure. Therefore, for any $f \in \mathcal{D}(A)$ and $g \in \mathcal{H}$ one has

$$
\langle A f, g\rangle=\int_{0}^{\infty} \xi d\left(P_{\xi} f, g\right),
$$

and hence the definition for a domain of $A$ becomes equivalent to

$$
\mathcal{D}(A)=\left\{f \in \mathcal{H}:\|A f\|^{2}:=\int_{0}^{\infty} \xi^{2} d\left(P_{\xi} f, f\right)<\infty\right\} .
$$

For any bounded Borel measurable function $\beta$ on the interval $(0, \infty)$, by the spectral theorem the operator $\beta(A)$ is bounded with $\|\beta(A)\|_{o p}=\|\beta\|_{\infty}$, and has an integral representation

$$
\beta(A):=\int_{0}^{\infty} \beta(\xi) d P_{\xi} .
$$

We say a vector (function) $f \in \mathcal{H}$ is a Paley-Wiener function with respect to the operator $A$ and belongs to $P W_{[a, b]}(A)$ for some $0 \leq a<b<\infty$ if $d\left(P_{\xi} f, f\right)$ is supported in the interval $[a, b]$. An equivalent definition of Paley-Wiener spaces $P W_{[a, b]}(\Delta)$ using the so-called functional form of the spectral theorem has been given in [1].

Associated to the operator $A$, there exist a measure $\nu$ on $\mathbb{R}^{+}$, a direct integral of Hilbert spaces $E=\left(E_{\lambda}\right)_{\lambda>0}$, and a unitary operator $\mathcal{F}$ which maps $\mathcal{H}$ onto $E$. The Hilbert space $E$ contains all $\nu$-measurable vector valued functions $\lambda \rightarrow e(\lambda) \in E_{\lambda}$ for which

$$
\|e\|_{E}=\left(\int_{0}^{\infty}\|e(\lambda)\|_{E_{\lambda}}^{2} d \nu(\lambda)\right)^{1 / 2}<\infty .
$$

It is natural to use the notation $E=\int_{0}^{\infty} E_{\lambda} d \nu(\lambda)$. The operator $\mathcal{F}$ transforms the domain of $A^{k}$ onto $E^{k}=\left\{e \in E \mid \lambda^{k} e \in E\right\}$. The norm on $E^{k}$ is given by

$$
\|e(\lambda)\|_{E^{k}}=\left(\int_{0}^{\infty} \lambda^{2 k}\|e(\lambda)\|_{E_{\lambda}}^{2} d \nu(\lambda)\right)^{1 / 2},
$$

and for any $f$ in the domain of $A^{k}, \mathcal{F}\left(A^{k} f\right)(\lambda)=\lambda^{k}(\mathcal{F} f)(\lambda)$. In analogy to the classical case, we call the unitary operator $\mathcal{F}$ the "Fourier transform" or "Plancherel transform" of $\mathcal{H}$, and $\nu$ the "Plancherel measure".

We say $f$ is "bandlimited" if its Fourier transform $\mathcal{F} f$ has support in $[a, b]$. It is easy to verify that $f$ belongs to the Hilbert space $P W_{[a, b]}(A)$ if and only if it is bandlimited. We say a function $f$ in $\mathcal{H}$ is "smooth" if it lies in $\bigcap_{k \in \mathbb{N}} \mathcal{D}\left(A^{k}\right)$. One can easily prove that $P W_{[a, b]}(A) \subset \bigcap_{k \in \mathbb{N}} \mathcal{D}\left(A^{k}\right)$, so every bandlimited function is smooth. 
Notation. In the sequel, we will drop the subscripts for the norms \|\|$_{o p},\|\|_{\infty}$, and \|\|$_{\mathcal{H}}$ when there is no confusion. And, we use $\preceq(\succeq)$ for $\leq(\geq)$ up to some constant.

\section{LitTlewood-PAley DeComposition}

In this section we will show how to decompose an abstract Besov function in terms of "smooth" and "bandlimited" functions. The smoothness and bandlimitedness of a function is understood in terms of the concepts described in the previous section. To begin with the idea, we need the following set up. Let $\hat{\psi}_{0}$ be a bounded and real valued function such that $\operatorname{supp}\left(\hat{\psi}_{0}\right) \subset[0,2]$ and $\hat{\psi}_{0}(0)=1$. Let $\hat{\psi}$ be another real valued function with $\operatorname{supp}(\hat{\psi}) \subset[1 / 2,2]$. For any $j \geq 1$, put $\hat{\psi}_{j}(\xi):=\hat{\psi}\left(2^{-j} \xi\right)$ and assume that the resolution of identity holds:

$$
\sum_{j \geq 0} \hat{\psi}_{j}(\xi)^{2}=1, \quad \text { a.e. } \xi \geq 0 .
$$

Note that for $j \geq 1, \hat{\psi}_{j}$ is compact supported and $\operatorname{supp}\left(\hat{\psi}_{j}\right) \subseteq\left[2^{j-1}, 2^{j+1}\right]$. By applying the spectral theorem for $A$ in (10), and under the assumptions that $\hat{\psi}_{0}$ and $\hat{\psi}$ are real valued, the following version of the Calderón decomposition, in complete analogy to the Euclidean space, holds:

$$
\sum_{j \geq 0} \hat{\psi}_{j}(A)^{2} f=f, \quad \forall f \in \mathcal{H}
$$

Here, the series converges in $\mathcal{H}$ and under our assumptions, the functions $\hat{\psi}_{j}(A) f$ are smooth and bandlimited with the "Fourier support" in $\left[2^{j-1}, 2^{j+1}\right]$.

Our main result in Theorem 3.1 presents a unified description of inhomogeneous abstract Besov spaces for all $\alpha>1 / 2$ in terms of smooth and bandlimited functions, as follows.

Theorem 3.1. Given any $\alpha>1 / 2,1 \leq q \leq \infty$ and $f \in \mathcal{H}$,

$$
\|f\|_{B_{q}^{\alpha}} \asymp\|f\|+\left(\sum_{j=0}^{\infty}\left(2^{j \alpha}\left\|\hat{\psi}_{j}(A) f\right\|\right)^{q}\right)^{1 / q},
$$

with the standard modifications for $q=\infty$, provided that both sides are finite.

By properties of the semigroup $\left\{T_{\tau}\right\}_{\tau>0}$ and that $\left\|\hat{\psi}_{j}(A)\right\|_{o p}=\left\|\hat{\psi}_{j}\right\|_{\infty} \leq 1$, one can easily prove the following estimations for the operator norm for all $r \in \mathbb{N}$ :

$$
\begin{aligned}
& \left\|\left(I-T_{\tau}\right)^{r} \hat{\psi}_{0}(A)\right\| \preceq \tau^{r}, \quad \text { and } \\
& \left\|\left(I-T_{\tau}\right)^{r} \hat{\psi}_{j}(A)\right\| \preceq \tau^{r} 2^{(j+1) r / 2} \quad j \geq 1 .
\end{aligned}
$$

The inequality constants are uniform in (4), i.e., they are independent of $j, r, \tau, \psi$, and $\psi_{0}$. We will use (44) in the following lemma to provide an upper estimation for the modulus of continuity $\Omega_{r}$. We need some preparation before the lemma: Let $m \in \mathbb{R}$ and $k<0$ such that $k+m \geq 0$. Put $w_{j}:=2^{k j}$ and $c_{j}:=2^{m j}$ for $j \in \mathbb{Z}$. 
Then,

Lemma 3.2. For $r \in \mathbb{N}, 1 \leq q<\infty$, and $f \in \mathcal{H}$,

$$
\Omega_{r}(s, f)^{q} \preceq s^{q r} \sum_{j=0}^{\infty}\left(2^{j r / 2} w_{j}^{1 / q} c_{j}\left\|\hat{\psi}_{j}(A) f\right\|\right)^{q} \quad \forall s \in(0,1) .
$$

The estimation holds for $q=\infty$ by modification.

Proof. Take $0<\tau \leq s<1$ and let $f \in \mathcal{H}$. By applying the discrete version of Calderon decomposition as in (2), for $f$ we have

$$
\left\|\left(I-T_{\tau}\right)^{r} f\right\| \leq \sum_{j=0}^{\infty}\left\|\left(I-T_{\tau}\right)^{r} \hat{\psi}_{j}(A)^{2} f\right\| .
$$

So,

$$
\begin{aligned}
\Omega_{r}(s, f)^{q}:=\left(\sup _{0<\tau \leq s}\left\|\left(I-T_{\tau}\right)^{r} f\right\|\right)^{q} & \leq\left(\sum_{j=0} \sup _{0<\tau \leq s}\left\|\left(I-T_{\tau}\right)^{r} \hat{\psi}_{j}(A)^{2} f\right\|\right)^{q} \\
& \leq\left(\sum_{j=0} w_{j} c_{j} \sup _{0<\tau \leq s}\left\|\left(I-T_{\tau}\right)^{r} \hat{\psi}_{j}(A)^{2} f\right\|\right)^{q} .
\end{aligned}
$$

The Hölder inequality and the relation (41) imply that

$$
\begin{aligned}
\Omega_{r}(s, f)^{q} & \preceq \sum_{j=0} w_{j}\left(c_{j} \sup _{0<\tau \leq s}\left\|\left(I-T_{\tau}\right)^{r} \hat{\psi}_{j}(A)^{2} f\right\|\right)^{q} \\
& \preceq \sum_{j=0}\left(w_{j}^{1 / q} c_{j}\left\|\hat{\psi}_{j}(A) f\right\|\right)^{q}\left(\sup _{0<\tau \leq s}\left\|\left(I-T_{\tau}\right)^{r} \hat{\psi}_{j}(A)\right\|\right)^{q} \\
& \preceq s^{q r} \sum_{j=0}\left(2^{j r / 2} w_{j}^{1 / q} c_{j}\left\|\hat{\psi}_{j}(A) f\right\|\right)^{q} .
\end{aligned}
$$

This proves the assertion of the lemma for $q>1$. For $q=1$ and $q=\infty$, the proof can be easily obtained by some modifications and similar arguments.

The decay property of $\Omega_{r}$ near the origin is a consequence of the preceding lemma as follows.

Corollary 3.3. For any $r \in \mathbb{N}$,

$$
\Omega_{r}(s, f)=\mathcal{O}\left(s^{r}\right) \quad 0<s<1 .
$$

\section{Proof of Theorem 3.1}

Theorem 3.1 is a result of Theorems 4.1 and 4.2

Theorem 4.1. Let $1 \leq q<\infty$ and $\alpha>1 / 2$. Given $f \in \mathcal{H}$, if $\left\{2^{j \alpha} \hat{\psi}_{j}(A) f\right\}_{j} \in$ $l^{q}\left(\mathbb{Z}^{+}, \mathcal{H}\right)$, then $f \in B_{q}^{\alpha}$ and

$$
\int_{0}^{1}\left(s^{-\alpha} \Omega_{r}(f, s)\right)^{q} d s / s \preceq \sum_{j \in \mathbb{Z}^{+}}\left(2^{j \alpha}\left\|\hat{\psi}_{j}(A) f\right\|\right)^{q},
$$

provided that the expression on the right is finite. The inequality also holds for $q=\infty$ with modification. 
Proof. Pick $r \in \mathbb{N}$ such that $r \leq 2 \alpha$. Let $k$ and $m$ be as above. Furthermore, assume that $k+m q \leq q(\alpha-r / 2)$. (Note that there is a large class of pairs $(k, m)$ such that $k+m \geq 0$ and satisfy the inequality.) Thus by Lemma 3.2 and $w_{j}=2^{k j}$ and $c_{j}=2^{m j}$, we obtain the following:

$$
\int_{0}^{1}\left(s^{-\alpha} \Omega_{r}(f, s)\right)^{q} d s / s \preceq \sum_{j=0}\left(2^{j r / 2} w_{j}^{1 / q} c_{j}\left\|\hat{\psi}_{j}(A) f\right\|\right)^{q} \preceq \sum_{j=0}\left(2^{j \alpha}\left\|\hat{\psi}_{j}(A) f\right\|\right)^{q} .
$$

This completes the proof of the theorem for $1 \leq q<\infty$. For $q=\infty$, the proof can be easily obtained by some modifications.

The next theorem completes the proof of Theorem 3.1

Theorem 4.2. Let $1 \leq q<\infty$ and $\alpha>1 / 2$. Given $f \in \mathcal{H}, r \in \mathbb{N}$ such that $r<2 \alpha$, we have

$$
\sum_{j \in \mathbb{Z}^{+}}\left(2^{j \alpha}\left\|\hat{\psi}_{j}(A) f\right\|\right)^{q} \preceq \int_{0}^{1}\left(s^{-\alpha} \Omega_{r}(f, s)\right)^{q} d s / s .
$$

The inequality also holds for $q=\infty$ with modification.

Proof. Let $u$ be the function that we had in Section 2, and $T_{s}=u(s A)$. For all $\lambda>0$, define

$$
G(\lambda):=\lambda^{-n} \int_{0}^{1} s^{-\alpha+r+1}(1-u(s \lambda))^{r} d s / s .
$$

Here, $n$ is a large number that can be fixed later. Therefore, by applying functional calculus theory for (8), for all $g \in \mathcal{H}$,

$$
G(A) g=\int_{0}^{1} s^{-\alpha+r+1} A^{-n}\left(I-T_{s}\right)^{r} g d s / s .
$$

If we take $g=\hat{\psi}_{j}(A) f$ in (9)), and use the Hölder inequality, we obtain the following inequalities:

$$
\begin{aligned}
& \left\|G(A) \hat{\psi}_{j}(A) f\right\| \leq \int_{0}^{1} s^{-\alpha}\left\|A^{-n}\left(I-T_{s}\right)^{r} \hat{\psi}_{j}(A) f\right\| s^{r+1} d s / s \\
& \quad \leq \int_{0}^{1} s^{-\alpha}\left\|\left(I-T_{s}\right)^{r} f\right\|\left\|A^{-n} \hat{\psi}_{j}(A)\right\| s^{r+1} d s / s \\
& \quad \leq\left(\int_{0}^{1}\left(s^{-\alpha}\left\|\left(I-T_{s}\right)^{r} f\right\|\right)^{q} d s / s\right)^{1 / q}\left(\int_{0}^{1} s^{(r+1) q^{\prime}}\left(2^{-n q^{\prime}(j-1)}\right) d s / s\right)^{1 / q^{\prime}} \\
& \quad \preceq 2^{-n(j-1)}\left(\int_{0}^{1}\left(s^{-\alpha} \Omega_{r}(s, f)\right)^{q} d s / s\right)^{1 / q} .
\end{aligned}
$$

Therefore, we proved that

$$
\left\|G(A) \hat{\psi}_{j}(A) f\right\|^{q} \leq 2^{-n(j-1) q} \int_{0}^{1}\left(s^{-\alpha} \Omega_{r}(s, f)\right)^{q} d s / s .
$$

From the other hand, with a simple calculation one can show that

$$
\left\|G(A) \hat{\psi}_{j}(A) f\right\| \succeq 2^{j(-n+1-\alpha+r)}\left\|\hat{\psi}_{j}(A) f\right\| .
$$


As a result of (10) and (11), we get

$$
\begin{aligned}
2^{j \alpha q}\left\|\hat{\psi}_{j}(A) f\right\|^{q} & \leq 2^{j \alpha q} 2^{-j q(-n+1-\alpha+r)}\left\|G(A) \hat{\psi}_{j}(A) f\right\|^{q} \\
& \preceq 2^{j(-2 n+1+r) q} \int_{0}^{1}\left(s^{-\alpha} \Omega_{r}(s, f)\right)^{q} d s / s .
\end{aligned}
$$

Hence,

$$
\sum_{j \geq 0} 2^{j \alpha q}\left\|\hat{\psi}_{j}(A) f\right\|^{q} \leq\left(\sum_{j \geq 0} 2^{j(-2 n+1+r) q}\right) \int_{0}^{1}\left(s^{-\alpha} \Omega_{r}(s, f)\right)^{q} d s / s .
$$

This proves the theorem if we let $n>\frac{r+1}{2}$.

Questions. These questions are still open and need to be answered. Can we remove the restriction $\alpha>1 / 2$ and prove Theorem 3.1 for $0<\alpha<1 / 2$ ? Can we prove Theorem 3.1 for $0<q<1$ ?

\section{ACKNOWLEDGEMENT}

The author would like to thank Professor Ronald R. Coifman for many helpful discussions and suggestions which improved the results of this paper.

\section{REFERENCES}

[1] M. Sh. Birman and M. Z. Solomjak, Spectral theory of selfadjoint operators in Hilbert space, Mathematics and its Applications (Soviet Series), D. Reidel Publishing Co., Dordrecht, 1987. Translated from the 1980 Russian original by S. Khrushchëv and V. Peller. MR.1192782 (93g:47001)

[2] Jens Gerlach Christensen and Gestur Ólafsson, Examples of coorbit spaces for dual pairs, Acta Appl. Math. 107 (2009), no. 1-3, 25-48, DOI 10.1007/s10440-008-9390-4. MR2520008 (2010h:43002)

[3] Jens G. Christensen, Azita Mayeli, and Gestur Ólafsson, Coorbit description and atomic decomposition of Besov spaces, Numer. Funct. Anal. Optim. 33 (2012), no. 7-9, 847-871, DOI 10.1080/01630563.2012.682134. MR2966135

[4] Ronald A. DeVore and George G. Lorentz, Constructive approximation, Grundlehren der Mathematischen Wissenschaften [Fundamental Principles of Mathematical Sciences], vol. 303, Springer-Verlag, Berlin, 1993. MR,1261635 (95f:41001)

[5] Hans G. Feichtinger and K. H. Gröchenig, Banach spaces related to integrable group representations and their atomic decompositions. I, J. Funct. Anal. 86 (1989), no. 2, 307-340, DOI 10.1016/0022-1236(89)90055-4. MR.1021139 (91g:43011)

[6] Hartmut Führ, Paley-Wiener estimates for the Heisenberg group, Math. Nachr. 283 (2010), no. 2, 200-214, DOI 10.1002/mana.200810164. MR2604118 (2011b:43014)

[7] Hartmut Führ and Azita Mayeli, Homogeneous Besov spaces on stratified Lie groups and their wavelet characterization, J. Funct. Spaces Appl., posted on 2012, Art. ID 523586, 41, DOI 10.1155/2012/523586. MR2923803

[8] Michael Frazier and Björn Jawerth, Decomposition of Besov spaces, Indiana Univ. Math. J. 34 (1985), no. 4, 777-799, DOI 10.1512/iumj.1985.34.34041. MR808825(87h:46083)

[9] Daryl Geller and Azita Mayeli, Continuous wavelets and frames on stratified Lie groups. I, J. Fourier Anal. Appl. 12 (2006), no. 5, 543-579, DOI 10.1007/s00041-006-6002-4. MR.2267634 (2007g:42055)

[10] Daryl Geller and Azita Mayeli, Continuous wavelets on compact manifolds, Math. Z. 262 (2009), no. 4, 895-927, DOI 10.1007/s00209-008-0405-7. MR.2511756 (2010g:42089)

[11] Daryl Geller and Azita Mayeli, Nearly tight frames and space-frequency analysis on compact manifolds, Math. Z. 263 (2009), no. 2, 235-264, DOI 10.1007/s00209-008-0406-6. MR2534117 (2011a:58050) 
[12] Daryl Geller and Azita Mayeli, Besov spaces and frames on compact manifolds, Indiana Univ. Math. J. 58 (2009), no. 5, 2003-2042, DOI 10.1512/iumj.2009.58.3741. MR2583490 (2011d:42082)

[13] Daryl Geller and Isaac Z. Pesenson, Band-limited localized Parseval frames and Besov spaces on compact homogeneous manifolds, J. Geom. Anal. 21 (2011), no. 2, 334-371, DOI 10.1007/s12220-010-9150-3. MR2772076 (2012c:43013)

[14] Einar Hille and Ralph S. Phillips, Functional analysis and semi-groups, American Mathematical Society Colloquium Publications, vol. 31, American Mathematical Society, Providence, R. I., 1957. rev. ed. MR0089373 (19,664d)

[15] S. Krein, I. Pesenson, Interpolation Spaces and Approximation on Lie Groups, The Voronezh State University, Voronezh, 1990.

[16] S. G. Krě̆n, Yu. İ. Petunīn, and E. M. Semënov, Interpolation of linear operators, Translations of Mathematical Monographs, vol. 54, American Mathematical Society, Providence, R.I., 1982. Translated from the Russian by J. Szücs. MR649411 (84j:46103)

[17] J.-L. Lions, Théorèmes de trace et d'interpolation. I, Ann. Scuola Norm. Sup. Pisa (3) 13 (1959), 389-403. MR0119092 (22 \#9858)

[18] Jaak Peetre, New thoughts on Besov spaces, Mathematics Department, Duke University, Durham, N.C., 1976. Duke University Mathematics Series, No. 1. MR0461123 (57 \#1108)

Department of Mathematics and Computer Sciences, City University of New York, Queensborough, Bayside, 222-05 56Th Avenue, New York, New York 11364

E-mail address: amayeli@qcc.cuny.edu 